\title{
AVALIAÇÃO DOS ATRIBUTOS FÍSICOS DE UM NITOSSOLO VERMELHO DISTROFÉRRICO SOB SISTE MA PLANTIO DIRETO, PREPARO CONVENCIONAL E MATA NATIVA(1)
}

\author{
Renato Lara de Assis ${ }^{(2)} \&$ Kléber Pereira Lanças ${ }^{(3)}$
}

\begin{abstract}
RESUMO
Com o aumento do tempo de uso do sistema plantio direto (SPD), atri butos físicos do solo são modificados; entretanto, os efeitos ainda não estão bem quantificados. $O$ presente estudo teve como objetivo avaliar o efeito do tempo de adoção do sistema plantio di reto, comparativamente com área de mata nativa e preparo convencional, usando atributos físicos do solo em um Nitossolo Vermelho distroférrico. Os sistemas de manejo estudados foram: mata nativa (MN), preparo convencional (PC), plantio direto com um ano (PD1), plantio direto com quatro anos (PD4), plantio direto com cinco anos (PD5) e plantio direto com 12 anos (PD12). Os valores de densidade do solo foram menores na profundidade de 0-5 cm em relação a 10-15 cm para todos os sistemas. Os sistemas de manejo MN e PD 12, na profundidade de 0-5 cm, apresentaram densidade do solo inferior à dos demais sistemas. 0 diâmetro médio geométrico (DMG) dos agregados do solo foi crescente com o tempo de adoção do sistema plantio direto, na profundidade de $0-5 \mathrm{~cm}$, e a mata nativa (MN) apresentou maior DMG em ambas as profundidades. $O$ tempo de adoção do SPD não promoveu diferenciação na porosidade total. O PD12 apresentou aumento da macroporosidade e di minuição da mi croporosidade na profundidade de 0-5 cm em relação aos demais sistemas. A resistência do solo à penetração não apresentou tendência predominante de variação com o tempo de adoção no SPD, tampouco em relação ao ti po de preparo. Os sistemas PD12 e MN apresentaram maiores velocidades de infiltração tridimensional de água no solo e condutividade hidráulica do solo saturado.
\end{abstract}

Temos de indexação: tempo de adoção, sistemas de manejo, qualidade do solo, estrutura.

\footnotetext{
(1) Parte da Tese de Doutorado em Energia na Agricultura, apresentada, pelo primeiro autor, à Faculdade de Ciências Agronômicas da Universidade Estadual Paulista - UNESP. Trabalho apresentado no XXIX Congresso Brasileiro de Ciência do Solo, Ribeirão Preto, 2003. Recebido para publicação em fevereiro de 2004 e aprovado em junho de 2005.

(2) Professor da Faculdade de Agronomia, Universidade de Rio Verde - FESURV. Fazenda Fontes do Saber, Caixa Postal 104, CEP 75901-970 Rio Verde (GO). E-mail: assis@esurv.br

(3) Professor do Departamento de Engenharia Rural, Universidade Estadual Paulista - FCA/UNESP. Caixa Postal 237, CEP 18603970 Botucatu (SP). E-mail: kplancas@fca.unesp.br
} 


\title{
SUMMARY: EVALUATION OF PHYSICAL ATTRIBUTES OF A DYSTROPHIC RED NITOSOL UNDER NO-TILLAGE, CONVENTIONAL TILLAGE AND NATIVE FOREST SYSTEMS
}

\begin{abstract}
As the time of adoption of no-tillage systems lengthens thee occur modification in soil physical attributes; however, these effects are not well quantified yet. Thepresent study had the objective to evaluate the effect of time of adoption of a no-tillage system, compared to native forest and a conventional tillage system using physical attributes of a Dystroferric Red Nitosol. Theeval uated systems were: PD1 (oneyear of adoption of no-tillage), PD4 (notillage for four years), PD5 (no-tillage for five years), PD12 (no-tillage for 12 years), one system under conventional tillage (PC for 18 years) and another without useor intervention (nativeforest-MN). Theincreasein no-tillageadoption timeled to a decreasein bulk density and compaction at the $0-5 \mathrm{~cm}$ depth, but cause no al terations at the $10-15 \mathrm{~cm}$ depth. The mean geometric diameter (DMG) of aggregates increased along the time of adoption of notillageat the $0-5 \mathrm{~cm}$ depth and the MN presented thelargest DMG at both soil depths. The time of adoption of the no-tillage system caused no differentiation in total soil porosity. Penetration resistancepresented no predominant trend of variation al ong thetimeof adoption of no-tillage. Therewas also notrend in relation to the other systems. PD12, at the depth of $0-5 \mathrm{~cm}$, presented thelargest macro and thesmallest micro porosity. Soil water infiltration was faster and thesaturated hydraulic conductivity of thesoil was higher under thesystems PD12 and MN.
\end{abstract}

Index terms: adoption time, management systems, soil quality, structure.

\section{INTRODUÇÃO}

Com o aumento do tempo de uso do sistema plantio direto (SPD), os atributos físicos dos solos têm sido modificados, necessitando, portanto, de pesquisas com períodos de duração mais longos para estudar os fenômenos ligados à sua estrutura. Efeitos sobre alguns atributos do solo já são reportados na literatura, tais como: a densidade do solo, que mostra tendência de aumento nos primeiros anos de cultivo e, com o passar dos anos, apresenta tendência de diminuição, o aumento da porosidade total e o aumento notamanho de agregados (Da Ros et al., 1997). Entretanto, muitas dúvidas sobre o sistema persistem, sendo necessário obter informações sobre outros atributos físicos.

A utilização intensiva de equipamentos agrícolas em todas as operações agrícol as (semeadura, tratos culturais e colheita) tem promovido aumento da compactação, principalmentena zona de exploração do sistema radicular da planta. A principal razão desse fenômeno é a repetitividade das operações realizadas ao longo dos anos (Oliveira, 2002). I números autores sugerem quea compactação do solo seja identificada, tanto por meio de atributos físicos, tais como: densidade do solo, distribuição de poros por tamanho eestabilidade deagregados emágua, ou como por meio da resistência do solo à penetração.

A compactação pelo tráfego de máquinas nas diferentes operações de preparo do sol o, semeadura, tratos culturais e col heita é constatada em diversos trabalhos (Tormena \& Rol off, 1996; Flowers \& Lal, 1998; Dias J unior et al., 1999 e Silva et al., 2000b). O comportamento do solo, conforme a aplicação de carga na superfície, depende das características do rodado e das propriedades físicas do solo. Nosistema plantio direto, o revolvimento do solo é restrito à linha de semeadura e, no entanto, o tráfego ocorre normal mente em toda extensão da área, resultando num aumento da compactação do solo, principalmente em superfície.

Estudando o efeito dos rodados do trator em três sistemas de preparo durante a semeadura, Sidiras \& Vieira (1984) observaram, nas faixas compactadas sob rodas, uma redução na infiltração de água e maiores val ores de densidade do sol o. A compactação da camada de semeadura pode ser limitante no processo de germi nação das sementes, contribuindo, por sua vez, para reduções da produtividade.

A condutividade hidráulica do solo saturado é considerada um dado de grande utilidade na diferenciação dos efeitos de sistemas de preparo na movimentação de água no perfil. A compactação do solo e a descontinuidade dos poros reduzem a condutividade hidráulica (Reichardt, 1996). O sistema plantio direto, por apresentar um sistema permanente de bioporos e canais deixados por raízes que apodrecem, permite uma melhor drenagem (Boone, 1988). Arzeno (1990) obteve valores de condutividade hidráulica do solo saturado nos sistemas plantio direto e escarificador cerca de duas vezes maior que no sistema de plantio convencional em Latossol o Vermel ho distroférrico. 
Diversos trabalhos experimentais relatam os efeitos benéficos dos sistemas conservacionistas no mel horamento das propriedades físicas do solo. A cobertura do solo com plantas ou seus resíduos reduz a temperatura máxima do solo e as perdas de água por evaporação (Bragagnolo \& Mielniczuk, 1990). Os resíduos de culturas também proporcionam um aumentona taxa de infiltração de água no sol o (Silva, 1986).

As maiores vel ocidades de infiltração de água no solo ocorre em sistema plantio direto, quando comparado ao sistema convencional (Derpsch et al., 1986; Roth et al., 1988; Arzeno, 1990; Castro, 1995), a cobertura reduz o efeito da desagregação, evitando - selamento superficial provocado pela obstrução dos poros por partículas finas desagregadas (Castro et al., 1987).

E márea de cerrado, por duas décadas em sistema plantio direto, Oliveira (2002) observou que o tráfego de máquinas e a ausência de revolvimento promoveram alterações na estrutura do solo, principalmente na macroporosi dade na profundidade de $0-5 \mathrm{~cm}$. Em Latossolo Vermel ho distrófico, Albuquerque et al. (1995) constataram que, ao final de seteanos, não ocorreram diferenças de densidade do solo, por osidade total, macro e microporosidade entre o sistema plantio direto e o preparo convencional.

O maior efeito agregante no sistema plantio direto em comparação ao sistema convencional na camada superficial foi relatado em diversos trabalhos: Carpenedo \& Mielniczuk, 1990; Da Ros et al., 1997; Silva et al., 2000a; Beutler et al., 2001; Oliveira, 2002.

Em plantio direto, ocorre um aumento do diâmetro médio geométrico (DMG) dos agregados, sendo esse aumento mais acentuado nos primeiros anos de instalação do sistema (Da Ros et al., 1996). Após cinco anos de cultivo, Da Ros et al. (1997) observaram que o DMG em plantio direto é estatisticamente equival ente ao do campo nativo.

Este trabalho teve por objetivo avaliar o efeito do tempo de adoção do sistema plantio direto, comparativamente com área de mata nativa e preparo convencional, sobre atributos físicos do solo.

\section{MATERIAL E MÉTODOS}

O estudo foi realizado em um $\mathrm{Nitossolo}$ Vermel ho distroférrico, relevo ondulado $\left(0-4 \mathrm{~cm} \mathrm{~m}^{-1}\right)$ textura muita argilosa, localizado nas áreas de produção e de experimentação da Fazenda Lageado no campus da UNESP - Botucatu (SP), utilizando os sistemas de manejo sob mata nativa (MN), preparo convencional (PC), plantio direto com um ano (PD1), plantio direto com quatro anos (PD4), plantio direto com cinco anos (PD5) e plantio direto com 12 anos (PD12). As áreas são descritas a seguir:

Mata nativa (MN) - Mantida há mais de 40 anos sem cultivo ou qualquer outro tipo de atividade antrópica. Localizada nas coordenadas 22 ० 48 ' 28 " S e $48^{\circ} 25^{\prime} 39^{\prime \prime}$ W.

Preparo convencional (PC) - A área foi cultivada com milho, desde 1983, utilizando a grade aradora e grade niveladora para o preparo do solo. Localizada nas coordenadas $22 \circ 48$ ' 19 " S e $48 \circ 25$ ' 41 "W.

Plantio direto com um ano (PD1) Correspondeà área de produção comercial da fazenda. Nas últimas duas safras, a área foi cultivada com soja e, anteriormente, com o milho. Localizada nas coordenadas $22^{\circ} 48^{\prime} 56^{\prime \prime} \mathrm{S}$ e $48^{\circ} 25^{\prime} 39^{\prime \prime} \mathrm{W}$.

Plantio direto com quatro anos (PD4) - Até o verão de 1996, a área era cultivada com a cultura do milho no sistema de preparo convencional, quando se instal ou o sistema plantio direto sobre os restos de cultura e vegetação espontânea. Coordenadas $22^{\circ} 49^{\prime} 3^{\prime \prime}$ S e $48^{\circ} 25^{\prime} 44^{\prime \prime} \mathrm{W}$.

Plantio di reto com cinco anos (PD5) - I niciouse no sistema plantio direto em 1996 com o milho, nas safras de 1996/1997; 1997/1998 e a soja, nas safras de 1998/1999; 1999/2000; 2000/2001, como culturas de verão. Coordenadas $22 \circ 48^{\prime} 25^{\prime \prime} \mathrm{S}$ e $48 \circ 25$ ' 40 "W.

Plantio direto com 12 anos (PD12) - N overão de 1988, o solo foi preparado mediante aração a aproximadamente $25 \mathrm{~cm}$ de profundidade, seguida de duas gradagens niveladoras, ea área foi cultivada com soja. Nos anos seguintes, a área foi utilizada da seguinte forma: De 1989 a 1998, cultivou-se a soja como cultura de verão. De 1999 a 2000, a área ficou em pousio no período de verão, apenas com o manejo da vegetação espontânea com a aplicação de herbicidas. Na safra de 2001/2002, foi cultivada com soja, como cultura de verão. Coordenadas $22^{\circ} 49^{\prime} 40$ " S e $48^{\circ} 25^{\prime} 39^{\prime \prime} \mathrm{W}$.

O tamanho de cada área experimental correspondente a cada sistema de manejo apresentava uma área de $1.000 \mathrm{~m}^{2}(50 \times 20 \mathrm{~m})$. Nestas áreas, foram identificadas três parcelas $(3 \times 3 \mathrm{~m})$, distribuídas aleatoriamente, onde foram realizadas três amostragens por parcela. Os pontos amostrais nas parcelas foram escol hidos na entrelinha da última cultura em cada sistema de plantio, procurando a melhor representatividade. Amostras foram col etadas nas profundidades de $0-5$ e $10-15 \mathrm{~cm}$.

Para determinar os limites deconsistência do solo (limites deliquidez, plasticidadee contração), foram col etadas amostras al eatoriamente, totalizando 36 amostras (seis sistemas $x$ duas profundidades $x$ três repetições). Os limites de consistência do solo seguiram métodos de Sowers (1965). 
A matéria orgânica, macro e microporosidade e densi dade do sol o foram determinadas de acordo com métodos da E mbrapa (1997). O cál culo do Diâmetro Médio Geométrico (DMG) dos agregados do sol o foi feito conforme Kemper \& Chepil (1965).

A resistência do solo à penetração foi determinada em diferentes pontos dentro de cada sistema de manejo até à profundidade de $40 \mathrm{~cm}$ (30 repetições por sistema), utilizando-se um penetrômetro hidráulico-eletrônico, construído por Lanças \& Santos (1998). Avaliou-se o índice de cone nas profundidades de 0-10, 10-20, 20-30 e 30-40 cm.

As medidas de infiltração da água no solo e de condutividade hidráulica do solo saturado foram realizadas no campo com o emprego do permeâmetro de fluxo constante (Permeâmetro de Guelph). Procedimentos de campo foram utilizados segundo recomendações de Lombardi Neto et al. (1993). A condutividade hidráulica do solo saturado foi medida na profundidade de $0-15 \mathrm{~cm}$, empregando-se duas cargas hidráulicas, de 6 e $9 \mathrm{~cm}$. Estas baseadas na recomendação para solos com textura argilosa.

A obtenção dos dados de condutividade hidráulica do solo saturado e infiltração da água no solo sob condições de saturação de campo foi realizada com o uso do software GPM.EXE (4).

Os dados experimentais foram analisados em delineamento inteiramente casualizado, incluindo a profundidade de amostragem como subfator.

Os resultados das análises físicas foram submetidos à análise de variância e teste de Tukey a $5 \%$ para a comparação de médias pel o programa SAS (Statistical Analysis System - versão 6.10 (SAS 1995)).

\section{RESULTADOS E DISCUSSÃO}

Os valores de densidade do sol o foram menores, com diferença estatisticamente significativa, na profundidade de $0-5 \mathrm{~cm}$ em relação a $10-15 \mathrm{~cm}$, para todos os sistemas de manejo (Quadro 1). Tal fato é devido ao teor de matéria orgânica nesta profundidade, o que favorece a diminuição da densidade do solo pela sua maior estruturação. 0 tempo de adoção no sistema plantio direto proporcionou diminuição na densidade do solo na profundidade de $0-5 \mathrm{~cm}$ e não causou al terações na densidade do solo na profundidade de $10-15 \mathrm{~cm}$.

Os sistemas MN ePD12, na profundidade de 0 $5 \mathrm{~cm}$, apresentaram densidade do solo significativamente diferente dos demais sistemas, evidenciando o efeito do tempo de utilização do

(4) Aplicativo computacional desenvolvido pelo pesquisador Dr. Sidney Rosa Vieira - Seção de Conservação do Solo - IAC sistema plantio direto na recuperação estrutural do solo.

A maior estabilidade de agregados no PD12 em relação aos demais sistemas sob plantio direto, verificada por meio do DMG (Quadro 1), pode ser explicada pelas práticas de manejo; a saber: preparo do solo, adição de materiais orgânicos e histórico cultural da área, que têm significativa influência sobre a agregação, quando considerados Iongos períodos de tempo. O DMG sob PD12 foi o que mais se aproximou do apresentado pel o sol o sob mata. A maior agregação no sistema $\mathrm{MN}$ é resultante do grande acúmulo de matéria orgânica ao longo dos anos, em virtude da não ação antrópica por mais de 40 anos, influenciando fortemente a agregação do solo. O DMG foi crescente com o tempo de adoção no sistema plantio direto, na profundidade de 0 $5 \mathrm{~cm}$, ea mata nativa (MN) apresentou maior DMG em ambas as profundidades.

Na profundidade de $0-5 \mathrm{~cm}$, o DMG teve um aumento da seguinte ordem: PD1 (75 \%), PD4 (77 \%), PD5 (243 \%), PD12 (366 \%) e M N (476 \%) em relação ao preparo convencional.

Com exceção do PD12, o tempo de adoção no sistema plantio direto não promoveu modificações na distribuição de poros por tamanho. O efeito da mobilização do solo no PC, favorecendo a formação de macroporos, não foi constatada no presente estudo. Observa-se que, no PD12, ocorreu um aumento da macroporosidade e diminuição da microporosidade na camada de $0-5 \mathrm{~cm}$ em relação ao preparo convencional. O tempo de adoção no sistema plantio direto não promoveu diferenciação na porosidade total. O PD12 na profundidade de 0$5 \mathrm{~cm}$ apresentou maior macroporosidade e menor micropor osidade em relação aos demais sistemas.

Os valores de umidade do solo no momento da avaliação da resistência do solo à penetração nos sistemas estudados (Quadro 2) encontram-se dentro, e, em alguns casos, pouco abaixo da faixa de friabilidade do solo (LP-LC) (Quadro 1). Segundo Torres \& Saraiva (1999), a faixa de friabilidade éa indicada para determinação da resistência do solo à penetração.

Os diferentes sistemas de cultivo e tempo de adoção no sistema plantio direto influenciaram a resistência do solo à penetração de forma mais acentuada, abaixo da camada de $0-10 \mathrm{~cm}$.

Os valores dos índices de cone (IC) encontrados neste estudo situam-se dentro da faixa de 0,7 a 3,5 MPa (Quadro 2), refletindo os resultados para o $I_{(0-10)}$, de maneira geral, os efeitos da mobilização, da maior quantidade de matéria orgânica e do tráfego em cada sistema de manejo. A resistência do solo à penetração não apresentou tendência predominante de variação com o tempo de adoção no sistema plantio direto e em relação ao tipo de preparo. 
Quadro 1. Atributos físicos e teor de matéria orgânica para os diferentes sistemas de manejo, profundidades e tempo de adoção do sistema plantio direto

\begin{tabular}{|c|c|c|c|c|c|c|c|c|c|}
\hline Sistema & Profundidade & DMG(1) & Ds(1) & Macro(1) & Micro(1) & $\mathbf{L L}^{(2)}$ & $\mathbf{L P}(2)$ & $L C(2)$ & MO(1) \\
\hline & $\mathrm{cm}$ & $\mathrm{mm}$ & $\mathrm{kg} \mathrm{dm}^{-3}$ & $\mathrm{dm}^{3}$ & $\mathrm{I}^{-3}$ & 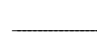 & $\mathrm{kg} \mathrm{kg}^{-1}$ & {[} & $\mathrm{g} \mathrm{kg}^{-1}$ \\
\hline \multirow[t]{2}{*}{$\mathrm{MN}$} & $0-5$ & 4,78 Aa & $1,05 \mathrm{Cb}$ & $0,19 \mathrm{Ba}$ & $0,38 \mathrm{Bb}$ & 0,58 & 0,46 & 0,26 & $105,2 \mathrm{Aa}$ \\
\hline & $10-15$ & 4,34 Ab & 1,22 Ca & $0,11 \mathrm{Ab}$ & $0,44 \mathrm{Aa}$ & 0,46 & 0,35 & 0,28 & $53,2 \mathrm{Ab}$ \\
\hline \multirow[t]{2}{*}{ PC } & $0-5$ & 0,83 Ea & $1,22 \mathrm{Ab}$ & $0,17 \mathrm{Ba}$ & $0,42 \mathrm{Ab}$ & 0,42 & 0,32 & 0,21 & 35,3 Ca \\
\hline & $10-15$ & $0,96 \mathrm{Ea}$ & 1,31 Ba & $0,11 \mathrm{Ab}$ & $0,45 \mathrm{Aa}$ & 0,43 & 0,34 & 0,20 & $37,4 \mathrm{ABa}$ \\
\hline \multirow[t]{2}{*}{ PD1 } & $0-5$ & $1,45 \mathrm{Db}$ & $1,17 \mathrm{ABb}$ & $0,22 \mathrm{ABa}$ & $0,39 \mathrm{ABb}$ & 0,43 & 0,33 & 0,22 & $39,1 \mathrm{BCa}$ \\
\hline & $10-15$ & $2,00 \mathrm{Ba}$ & $1,35 \mathrm{ABa}$ & $0,13 \mathrm{Ab}$ & $0,44 \mathrm{Aa}$ & 0,43 & 0,35 & 0,22 & $37,7 \mathrm{ABa}$ \\
\hline \multirow[t]{2}{*}{ PD 4} & $0-5$ & 1,47 Da & $1,17 \mathrm{ABb}$ & $0,17 \mathrm{Ba}$ & $0,42 \mathrm{ABa}$ & 0,44 & 0,33 & 0,25 & $39,9 \mathrm{BCa}$ \\
\hline & $10-15$ & $1,31 \mathrm{DEb}$ & 1,36 Aa & $0,13 \mathrm{Ab}$ & $0,43 \mathrm{Aa}$ & 0,45 & 0,35 & 0,28 & $34,1 \mathrm{Bb}$ \\
\hline \multirow[t]{2}{*}{ PD5 } & $0-5$ & $2,85 \mathrm{Ca}$ & $1,12 \mathrm{Bb}$ & $0,18 \mathrm{Ba}$ & $0,40 \mathrm{ABb}$ & 0,43 & 0,32 & 0,28 & $48,3 \mathrm{Ba}$ \\
\hline & $10-15$ & $1,89 \mathrm{BCb}$ & $1,34 \mathrm{ABa}$ & $0,11 \mathrm{Ab}$ & $0,43 \mathrm{Aa}$ & 0,43 & 0,34 & 0,29 & $36,6 \mathrm{ABb}$ \\
\hline \multirow[t]{2}{*}{ PD12 } & $0-5$ & 3,87 Ba & $1,06 \mathrm{Cb}$ & $0,27 \mathrm{Aa}$ & $0,34 \mathrm{Cb}$ & 0,35 & 0,28 & 0,27 & $43,1 \mathrm{BCa}$ \\
\hline & $10-15$ & $1,54 \mathrm{CDb}$ & $1,35 \mathrm{ABa}$ & $0,13 \mathrm{Ab}$ & $0,39 \mathrm{Ba}$ & 0,35 & 0,28 & 0,26 & $28,8 \mathrm{Bb}$ \\
\hline
\end{tabular}

$\overline{\mathrm{DMG}}=$ diâmetro médio geométrico; Ds = densidade do solo; Macro = macroporosidade; Micro = microporosidade; LL =limite de liquidez; LP = limite de plasticidade; LC = limite de contração e $\mathrm{MO}=$ matéria orgânica do solo.

MN: Mata Nativa; PC: Preparo Convencional; PD1: Plantio Direto com um ano; PD4: Plantio Direto com quatro anos; PD5: Plantio Direto com cinco anos; PD12: Plantio Direto com 12 anos.

Letras maiúsculas, na coluna, comparam diferenças entre os sistemas para cada profundidade, a $5 \%$ pelo teste Tukey.

Letras minúsculas, na coluna, comparam diferenças entre as duas profundidades de cada sistema, a $5 \%$ pelo teste Tukey.

(1) Média de nove repetições. (2) Média de três repetições.

Quadro 2. Valores médios dos índices de cone (IC) nas diferentes profundidades para os diferentes sistemas de manejo e tempo de adoção do sistema plantio direto

\begin{tabular}{|c|c|c|c|c|}
\hline Sistema(1) & $I_{0-10}$ & $I C_{10-20}$ & $I C_{20-30}$ & $I C_{30-40}$ \\
\hline & \multicolumn{4}{|c|}{$\mathrm{MPa}$} \\
\hline $\mathrm{MN}$ & $0,65 \mathrm{Cc}$ & $1,91 \mathrm{Cb}$ & 3,17 Ba & 3,37 Aa \\
\hline PC & $1,15 \mathrm{Abc}$ & 1,92 Ca & $1,60 \mathrm{Db}$ & $1,12 \mathrm{DC}$ \\
\hline PD1 & $0,74 \mathrm{Cc}$ & $2,06 \mathrm{Cb}$ & $2,80 \mathrm{Ca}$ & $2,73 \mathrm{Ba}$ \\
\hline PD4 & $1,27 \mathrm{Ac}$ & $3,07 \mathrm{ABb}$ & 3,47 ABa & $3,25 \mathrm{Aab}$ \\
\hline PD5 & $1,17 \mathrm{ABb}$ & 3,39 Aa & 3,53 Aa & 3,48 Aa \\
\hline PD12 & $1,05 \mathrm{BC}$ & $2,76 \mathrm{Ba}$ & 2,53 Ca & $2,06 \mathrm{Cb}$ \\
\hline
\end{tabular}

Letras maiúsculas, na coluna, comparam diferenças entre os sistemas a $5 \%$ pelo teste Tukey.

Letras minúsculas, na linha, comparam diferenças entre as profundidades (IC) para cada sistema a $5 \%$ pelo teste Tukey.

(1) Média de nove repetições.

Nos diferentes sistemas de cultivo, os sistemas PD4 e PD5 foram os que apresentaram maiores valores de (IC) em todas as profundidades, sendo esses valores, na profundidade de 20 a $30 \mathrm{~cm}$, da ordem de 3,5 MPa.

Adotando o valor de 2,5 MPa como resistência crítica ao crescimento radicular da soja na consistência friável, segundo Torres \& Saraiva
(1999), os resultados indicam que as áreas de PD4, PD5 e PD12, nas profundidades de 10-20 e 20$30 \mathrm{~cm}$, apresentam val ores superiores, evidenciando possíveis restrições à penetração de raízes, em períodos de déficit hídrico.

Os resultados apresentados (Quadro 2) indicam ser a resistência do sol o à penetração determinação recomendável para avaliar a compactação, 
considerando os sistemas de manejo e intensidade de tráfego, desde que suas determinações sejam realizadas com o solo dentro de uma mesma faixa de umidade, aumentando, desta forma, a sensibilidade das avaliações.

A análise de variância mostrou efeitos significativos do manejo e tempo de adoção no sistema plantio direto sobre a infiltração tridimensional e condutividade hidráulica do solo saturado (Quadro 3). Os sistemas PC, PD1, PD4 e PD5 não diferiram estatisticamente em relação à infiltração tridimensional, fato explicado, possivel mente, pela igual dade na macroporosidade (Quadro 1).

Os baixos val ores de condutividade hidráulica no plantio convencional (PC) em relação ao PD12 (Quadro 3) deveram-se à desestruturação excessiva do solo, em decorrência da utilização da aração e gradagem, resultando, assim, em grandequantidade de partículas finas, selando parcialmente os poros, dificultando a ocorrência de maiores taxas de infiltração. O PD12 e a MN destacaram-se quanto aos atributos analisados. A condutividadehidráulica noPD12 eno MN foi 15 e 11 vezes, respectivamente, maiores que o PC (Quadro 3). Arzeno (1990), em estudo com permeâmetro de Guelph, encontrou também resultados superiores do plantio direto em relação ao PC. A explicação possível deve-se à continuidade de poros, à tortuosidade dos interstícios e à maior atividade biológica que facilita a movimentação tridimensional da água, dados também encontrados por Chan \& Heenan (1993),

\section{Quadro 3. Médias geométricas(1) de infiltração tridimensional correspondente à segunda carga hidráulica $(9 \mathrm{~cm})$ e da condutividade hidráulica do solo saturado e seus respectivos coeficientes de variação, determinados na profundidade de $0-15 \mathrm{~cm}$ nos diferentes sistemas de manejo e tempo de adoção do sistema plantio direto}

\begin{tabular}{lcc}
\hline Sistema & $\begin{array}{c}\text { Infiltração } \\
\text { tridimensional }\end{array}$ & $\begin{array}{c}\text { Condutividade } \\
\text { hidráulica }\end{array}$ \\
\hline & \multicolumn{2}{c}{$\mathrm{mm} \mathrm{h}^{-1}$} \\
\hline MN & $233,45^{(1)} \mathrm{B}$ & $119,18^{(1)} \mathrm{A}$ \\
PC & $83,50 \mathrm{C}$ & $10,42 \mathrm{C}$ \\
PD1 & $94,82 \mathrm{C}$ & $16,67 \mathrm{BC}$ \\
PD4 & $105,65 \mathrm{C}$ & $24,59 \mathrm{~B}$ \\
PD5 & $91,97 \mathrm{C}$ & $18,33 \mathrm{BC}$ \\
PD12 & $374,63 \mathrm{~A}$ & $157,51 \mathrm{~A}$ \\
CV $(\%)$ & 6,54 & 30,13 \\
\hline
\end{tabular}

Letras maiúsculas, na coluna, comparam diferenças entre os sistemas a $5 \%$ de probabilidade pelo teste Tukey.

(1) Média de 30 repetições por sistema.
Castro (1995) e Sarvasi (1994). Estimativas de condutividade hidráulica saturada e infiltração, estatisticamente superiores para o plantio di reto em relação aos sistemas de preparo convencional podem estar relacionadas com o fato de este atributo sofrer grande influência da porosidade do solo, da matéria orgânica e da estruturação do solo, conforme argumentam Cadima et al. (1980) eJ abro (1992). É de se esperar que, no plantio direto, as condições gerais dos atributos do solo estejam mais próximas das condições naturais que no plantio convencional.

A infiltração tridimensional no sistema $M N$ foi menor do que no PD12 (Quadro 3), e al guns autores encontraram maior infiltração em área de mata nativa do que em solos cultivados (Leite \& Medina,1984; Corrêa, 1985), resultados estes não observados neste estudo, provavelmente pela presença de maior macroporosidade no PD12 (Quadro 1) na profundidade de $0-5 \mathrm{~cm}$.

Os valores de condutividade hidráulica foram classificados segundo diferentes classes de permeabilidade adaptadas do Soil Survey Staff (1993), como: PC, PD1 e PD5: lenta (5-20 $\mathrm{mm} \mathrm{h}^{-1}$ ), PD4: lenta a moderada (20-63,5 $\left.\mathrm{mm} \mathrm{h}^{-1}\right), \mathrm{MN}$ : moderada (63,5-127 $\mathrm{mm} \mathrm{h}^{-1}$ ) e o PD12: moderada a rápida (127-254 $\left.\mathrm{mm} \mathrm{h}^{-1}\right)$.

Nesteestudo, foram encontrados val ores elevados de coeficiente de variação (CV) de 30 \% para a condutividade hidráulica do sol o saturado com o permeâmetro de Guelph (Quadro 3), concordando com trabalhos de Arzeno (1990), Sarvasi (1994), Guimarães (2000) e Beutler et al. (2001). Housseini et al. (1993) encontraram valores de coeficiente de variação acima de 78 \%. Castro (1995) obteve coeficientes de variação de 13,74 a 33,14 \%, para a infiltraçãotridimensional, e de 14,75 a $23,60 \%$, para a condutividade hidráulica em diferentes profundidades.

\section{CONCLUSÕES}

1. O tempo de adoção do sistema plantio direto proporcionou diminuição na densidade do solo na profundidade de $0-5 \mathrm{~cm}$ e não causou alterações na densidade do sol o na profundidade de $10-15 \mathrm{~cm}$.

2. O DMG foi crescente com o tempo de adoção do sistema plantio direto na profundidade de $0-5 \mathrm{~cm}$ e a MN apresentou maior DMG em ambas as profundidades.

3. A resistência do solo à penetração não apresentou tendência predominante de variação com o tempo de adoção do sistema plantio direto e em relação ao tipo de preparo.

4. O tempo de adoção do sistema plantio direto não promoveu diferenciação na porosidade total. 0 PD12 na profundidade de $0-5 \mathrm{~cm}$ apresentou mai or macroporosidade e menor microporosidade. 
5. Os tratamentos PD12 e MN apresentaram maiores velocidades de infiltração tridimensional de água no solo e condutividade hidráulica do solo saturado.

\section{AGRADECIMENTO}

À CAPES/PICDT, pela concessão da bolsa durante o curso de Doutorado.

\section{LITE RATURA CITADA}

ALBUQUERQUE, J.A.; REINERT, D.J .; FIORIN, J .E.; RUEDELL, J .; PETRERE, C. \& FONTINELLI, F. Rotação de culturas e sistemas de manejo do solo: Efeito sobre a forma da estrutura do solo ao final de sete anos. R. Bras. Ci. Solo, 19:115-119, 1995.

ARZENO, J.L. Avaliação física de diferentes manejos de solo em Latossolo Roxo distrófico. Piracicaba, Escola Superior de Agricultura Luiz de Queiroz, 1990. 259p. (Tese de Doutorado)

BEUTLER, A.N.; SILVA, M.L.N.; CURI, N.; FERREIRA, M.M.; CRUZ, J.C. \& PEREIRA FILHO, I.A. Resistência à penetração e permeabilidade de Latossolo Vermelho distrófico típico sob diferentes sistemas de manejo na região dos cerrados. R. Bras. Ci. Solo, 25:167-177, 2001.

BOONE, F.R. Weather and other environmental factors influencing crop responses to tillage and traffic. Soil Till. Res., 11:283-324, 1988.

BRAGAGNOLO, N. \& MIELNICZUK, J. Cobertura do solo por resíduos de oito seqüências de culturas e seu relacionamento com a temperatura e umidade do solo, germinação e crescimento inicial do milho. R. Bras. Ci. Solo, 14:91-98, 1990.

CADIMA, Z.A.; LIBARDI, P.L. \& REICHARDT, K. Variabilidade espacial da condutividade hidráulica em um Latossolo Vermelho-Amarelo textura média, no campo. R. Bras. Ci. Solo, 4:63-66, 1980

CARPENEDO, V. \& MIELNICZUK, J. Estado de agregação e qualidade de agregados de Latossol os Roxos, submetidos a diferentes sistemas de manejo. R. Bras. Ci. Solo, 14:99105, 1990.

CASTRO, O.M. Comportamento físico e químico de um Latossolo Roxo em função do seu preparo na cultura do milho (Zea mays L.). Piracicaba, Escola Superior de Agricultura Luiz de Queiroz, 1995. 174p. (Tese de Doutorado)

CASTRO, O.M.; VIEIRA, S.R. \& MARIA, I.C. Sistemas de preparo do solo e disponibilidade de água. In: SIMPÓSIO SOBRE MANEJ O DE ÁGUA NA AGRICULTURA, 1987, Campinas. Anais. Campina, Fundação Cargill, 1987. p.2751.

CHAN, K.Y. \& HEENAN, D.P. Surface hydraulic properties of a Red Earth under continuous cropping with different management practices. Aust. J . Soil Res., 31:13-24, 1993.
CORRÊA, J .C. Características físicas de um Latossolo Amarelo muito argiloso (Typic Acrorthox) do Estado do Amazonas, sob diferentes métodos de preparo do solo. Pesq. Agropec. Bras., 20:1381-1387, 1985.

DA ROS, C.O.; LOPES, C.E.L.; SECCO, D. \& PASA, L. Influência do tempo de adoção no sistema plantio direto nas características físicas de um Latossolo Vermelho-Escuro. In: REUNIÃO SUL-BRASI LEIRA DE CIÊNCIA DO SOLO, 1., Lages, 1996. Resumos. Pelotas, Sociedade Brasileira de Ciência do Solo - Núcleo Regional Sul, 1996. p.153-155.

DA ROS, C.O.; SECCO, D.; FIORIN, J.E.; PETRERE, C.; CADORE, M.A. \& PASA, L. Manejo do solo a partir de campo nativo: Efeito sobre a forma de estabilidade da estrutura ao final de cinco anos. R. Bras. Ci. Solo, 21:241247, 1997.

DERPSCH, R.; SIDIRAS, N. \& ROTH, C.H. Results of studies made from 1977 to 1984 to control erosion by cover crops and tillage techniques in Paraná, Brazil. Soil Till. Res., 8:253-263, 1986.

DIAS J UNIOR, M.S.; FERREIRA, M.M.; FONSECA, S.; SILVA, A.R. \& FERREIRA, D.F. Avaliação quantitativa da sustentabilidade estrutural dos sol os em sistemas florestais na região de Aracruz-ES. R. Árvore, 23:371-380, 1999.

EMPRESA BRASILEIRA DE PESQUISA AGROPECUÁRIA EMBRAPA. Centro Nacional de Pesquisa de Solos. Manual de métodos de análise de solo. 2.ed. Rio de J aneiro, 1997. $212 p$.

FLOWERS, M.D. \& LAL, R. Axle load and tillage effects on soil physical properties and soybean grain yield on a Mollic Ochraqualf in Northwest Ohio. Soil Till. Res., 48:21-35, 1998.

GUIMARÃES, E.C. Variabilidade espacial de atributos de um Latossolo Vermelho-Escuro textura argilosa da região do cerrado, submetido ao plantio direto e ao plantio convencional. Campinas, Universidade Estadual de Campinas, 2000. 89p. (Tese de Doutorado)

HOUSSEINI, E.; GALLICHAND, J . \& CARON, J . Comparison of several interpolators for smoothing hydraulic conductivity data in South West I ran. Am. Soc. Agric. Eng., 36:1687-1693, 1993.

J ABRO, J.D. Estimation of saturated hydraulic conductivity of soils from particle size distribution and bulk density data. Am. Soc. Agric. Eng., 35:557-560, 1992.

KEMPER, W.D. \& CHEPIL, W.S. Size distribuition of agregates. In: BLACK, C.A., ed. Methods of soil analysis: Physical and mineralogical properties including statistics of measurement and sampling. Madison, American Society of Agronomy, 1965. Part 1, p.499-510.

LANÇAS, K.P. \& SANTOS, C.A. Penetrômetro hidráulicoel etrônico equipado com DGPS para avaliação da compactação do solo. In: BALBUENA, R.H.; BENEZ, S.H. \& J ORAJ URIA, D., eds. Ingeniería rural y mecanización agraria en el ámbitolatinoamericano. La Plata, 1998. p.570576.

LEITE, J .A. \& MEDINA, B.F. Efeito de sistemas de manejo sobre as propriedades físicas de um Latossolo Amarelo do Amazonas. Pesq. Agropec. Bras., 19:1417-1422, 1984. 
LOMBARDI NETO, F.; CASTRO, O.M.; DECHEN, S.C.F.; VIEIRA, S.R. \& DE MARIA, I.C. Manual de coleta de amostras e análises físicas para fins de experimentação em conservação do solo. Campinas, Instituto Agronômico de Campinas, Seção de Conservação do Solo, 1993. 77p.

OLIVEIRA, G.C. Alterações estruturais e comportamento compressivo de um Latossolo submetido a diferentes sistemas de manejo por 20 anos no cerrado. Lavras, UniversidadeFederal deLavras, 2002. 78p. (TesedeDoutorado)

REICHARDT, K. Dinâmica da matéria e da energia em ecossistemas. 2.ed. Piracicaba, Escola Superior de Agricultura Luiz de Queiroz, 1996. 505p.

ROTH, C.H.; MEYER, B.; FREDE, M.G. \& DERPSCH, R. Effect of mulch rates and tillagesystems on infiltrability and other soil physical properties of an Oxisol in Paraná, Brazil. Soil Till. Res., 11:81-91, 1988.

SARVASI, F.O.C. Dinâmica da água, erosão hídrica e produtividade das culturas em função do preparo do solo. Piracicaba, Escola Superior deAgricultura Luiz deQueiroz, 1994. 147p. (Tese de Doutorado)

SAS Institute. SAS Ianguage and prodecure: usage. Version 6. Cary, 1995. 373p.

SIDIRAS, N. \& VIEIRA, M.J . Comportamento de um Latossolo Roxo Distrófico, compactado pelas rodas do trator na semeadura - rendimentos de três culturas. Pesq. Agropec. Bras., 19:1285-1293, 1984.
SILVA, H.P. I nfiltração de água em um Podzólico VermelhoEscuro fisicamente degradado em processo de recuperação. Porto Alegre, Universidade Federal do Rio Grande do Sul, 1986. 105p. (Tese de Mestrado)

SILVA, V.R.; REINERT, D.J . \& REICHERT, J .M. Resistência mecânica do solo à penetração influenciada pel o tráfego de uma colhedora em dois sistemas de manejo do solo. Ci. Rural, 30:795-801, 2000b.

SILVA, M.L.N.; CURI, N. \& BLANCANEAUX, P. Sistemas de manejo e qualidade estrutural de Latossolo Roxo. Pesq. Agropec. Bras., 35:2485-2492, 2000a.

SOIL SURVEY STAFF. Soil survey manual. Washington, USDA, 1993. 437p. (Handbook, 18)

SOWERS, G.F. Consistency. In: BLACK, C.A., ed. Methods of soil analisys physical and mineralogical properties including statistics of measurements and sampling. Madison, Americam Society of Agronomy, 1965. Part 1, p.391-399.

TORMENA, C.A. \& ROLOFF, G. Dinâmica da resistência à penetração de um solo sob plantio direto. R. Bras. Ci. Solo, 20:333-339, 1996.

TORRES, E. \& SARAIVA, O.F. Camadas de impedimento do solo em sistemas agrícolas com a soja. Londrina, Empresa Brasileira de Pesquisa Agropecuária, 1999. 58p. (Circular Técnica, 23) 